\title{
LA ÉTICA JURÍDICA EN EL BRAHMANISMO
}

\author{
Armando Alvares GARCIA JÚNIOR*
}

\begin{abstract}
* Profesor de derecho internacional público, derecho del comercio internacional y sistemas jurídicos no occidentales (grado en derecho), estructura económica mundial, negocios internacionales y dirección estratégica y política de la empresa - I (grado ADE), dirección estratégica y política de la empresa - II (curso de adaptación ADE) y negociación y marketing internacional (MBA-ADE) en la Universidad Internacional de la Rioja, España. Miembro del Grupo de Investigación "Culturas, religiones y derechos humanos en la sociedad actual" de la Universidad Internacional de la Rioja (GDI-I).

armando.alvares@unir.net
\end{abstract}

Recebido em: 25/03/2014 - Aprovado em: 17/06/2015 - Disponibilizado em: 15/07/2015

RESUMEN: El artículo analiza el tema de la ética financiera bajo la perspectiva del brahmanismo, alma mater de la compleja sociedad hindú. Las castas sociales y las fuentes dese peculiar derecho son también apreciados en la investigación.

PALABRAS CLAVE: Brahmanismo, derecho, castas, ética financiera, justicia.

\section{LEGAL ETHICS IN BRAHMINISM}

ABSTRACT: The article analyses the issue of financial ethics from the perspective point of view of Brahmanism, alma mater of the complex Hindu society. The social castes and the sources of that special law are also appreciated in the investigation.

KEYWORDS: Brahmanism, law, castes, financial ethics, justice.

\section{INTRODUCCCIÓN}

En el Brahmanismo, la ética financiera está estrechamente relacionada con la división social en castas. La progresiva laicización del derecho hindú (un crisol constituido por: a) derecho brahmánico, b) derecho islámico, c) costumbres antiquísimas y d) Common Law) y la abolición formal del sistema de castas resultante de la reforma legislativa de los años cincuenta del siglo pasado, no han logrado extinguir, en la práctica, la división de la sociedad india en cuatro castas y miles de intercastas y subcastas. De hecho, esa división es milenaria. El más antiguo texto sagrado de los hindús, el Rig-veda, establecía las siguientes grandes categorías (varnas): a) Bracmanes o Brahmanes (hasta hoy constituyen la casta de los sacerdotes ritualísticos, maestros y recitadores de las Escrituras Sagradas), b) Chatryas o Chatrías (de donde proceden los nobles, políticos y militares), c) Vaisyas o Vaishias (constituida por 
agricultores, ganaderos, mercaderes

y pequeños comerciantes) y d)

Sudras o Shudrás (constituida por campesinos y obreros).

A margen de estas castas (también consideradas varnas por el Código Manu) y de todas las intercastas y subcastas existentes, existe un grupo especial denominado "los intocables" (Dalits). Sus miembros son considerados impuros $\mathrm{y}$ socialmente tan bajos que ni siquiera tienen una casta (descastados). La abolición formal de las castas ha permitido a los niños dalits acceder a los colegios públicos. No obstante, por el repudio social que sufren, ellos son obligados a limpiar los váteres de los colegios donde estudian. También están prohibidos de beber agua en los grifos del colegio para no transmitir y contaminar con su karma a toda el agua de las tuberías y así, por extensión, macular el karma de los demás (estudiantes, profesores, empleados del colegio). La persona de casta superior eventualmente tocada por un dalit (o por su sombra) debe realizar un ritual de purificación. Algo similar ocurre con el contacto físico (o de su sombra) de un shudrá con un brahmán. El dharma de ambos se perjudica irremediablemente. Ellos no tienen derecho a nada y no pueden exigir nada a los miembros de cualquier casta, subcasta o intercasta. Como los oficios en India son asignados de acuerdo con la casta, los dalits ejecutan las tareas que nadie más desea realizar: lavaderos de ropas, poceros (obligados a retirar excrementos ajenos con las manos o pequeños cuencos), recogedores de cadáveres de animales, limpiadores de baños y drenajes etc. Como mencionamos, ellos no tienen ningún derecho. $\mathrm{Ni}$ mismo a recibir la paga por sus trabajos. Esperan recibirla pero, si eso no ocurre, no pueden exigir absolutamente nada.

Las personas pertenecientes a cada una de estas castas deben vivir separadamente. No pueden juntarse y mucho menos casarse. El matrimonio con una persona de inferior varna es considerado impuro y contaminante. También aceptar alimentos tocados o cocinados por una persona de casta inferior es considerado contaminante. Comer aún más, evidentemente. La explicación fundamentada en el hinduismo es 
que el alma es eterna y la casta en que la persona nazca es el resultado de las acciones practicadas en su vida anterior. De ese modo, la persona debe vivir en su categoría correspondiente, como forma de expiación, hasta el día de la muerte de su cuerpo físico. Solamente se concebiría el cambio de varna (categoría/casta) en su reencarnación, jamás durante su vida terrenal (hermetismo). Así, en función de los actos practicados durante la vida $-\mathrm{y}$ de haber aceptado resignadamente su destino como el resultado de su vida anterior - podría el alma subir o bajar de categoría en la siguiente reencarnación o hasta mismo reencarnar en el cuerpo de un animal (más bajo aún)

\section{LA ÉTICA EN EL BRAHMANISMO}

Hablar sobre ética financiera en el marco del brahmanismo es muy difícil porque, bajo la perspectiva occidental, no parece existir cualquier asociación con la moralidad pública o la justicia social. Esa situación descompensada se revela también en otros campos. Por ejemplo, en el ámbito del derecho penal,
Mayne $(1953)^{1}$ señala, en relación a la casta Shudrá, que: a) si agrede a cualquier persona de una casta superior, el miembro que ha usado para golpear debe ser amputado, b) si es hospede, debe trabajar para pagar la hospitalidad, c) está prohibido de acceder a las escrituras religiosas, d) caso escuche la recitación de los Vedas, sus orejas deben ser llenadas con plomo derretido, e) si es capaz de recitar la oración, su lengua debe ser cortada, f) si es capaz de memorizarla, debe ser descuartizado.

En un contexto como ese, señalar que los shudrás o los dalits paguen más o menos intereses por un préstamo es casi irrisorio, puesto que ellos están sometidos a brutales injusticias permanentemente. Tampoco se puede argumentar que su desprestigio social viola el derecho. Dos argumentos básicos pueden ser invocados a este respecto: 1) la palabra "derecho" no encuentra correspondencia en la lengua hindú. Lo más aproximado sería el término dharma (proveniente del sánscrito) que significa el orden natural $y$ social que regula el universo (macrocosmos) y los hombres (microcosmos). En la práctica, el derecho escrito tiene poco peso ante

\footnotetext{
${ }^{1}$ Mayne, John Dowson. (1953). Treatise on Hindu Law and Usage. Madras: Higginbothams.
} 
unas costumbres profundamente arraigadas en la sociedad y 2) son los propios textos sagrados para los hindúes que establecen esa división en castas. Las sanciones impuestas a las personas que violen las reglas que plasman el "orden natural y social" pueden ser tanto de naturaleza terrenal (multas, castigos corporales, restricción de derechos y privación de libertad) como espiritual (reencarnar, por ejemplo, como un cerdo, una rata, un cisne, una perdiz, una rana, un gato, un jabalí, un león, un tigre, un camello, un perro, una cabra, una oveja, un erizo, un puercoespín, un oso, un toro, un lobo, un asno, un ratón almizclero, un cocodrilo, un mono, un pájaro carnívoro, un buitre, un pavo real, un chataka $^{2}$, un insecto, un abejón, una langosta, un gusano, una hierba, un matorral, una liana, un bruto, un descastado chándala ${ }^{3}$, un pukkasa ${ }^{4}$, un Ulkamuka $^{5}$, un Kataputana ${ }^{6}$, un

\footnotetext{
2 Es el pájaro conocido como "cuclillo".

${ }^{3}$ Hijo de padre shudrá y madre bráhmana (o hija de un sacerdote brahmán). Viswanatha, S. V. (2013). Racial synthesis in Hindu culture (no. 47). London: Routledge.

${ }^{4}$ Hijo de un shudrá con un nisada (por su vez hijo de un brahmán con un shudrá). Viswanatha, S. V. (2013). Racial synthesis in Hindu culture (no 47). London: Routledge.

${ }^{5}$ Espíritu que se alimenta de lo que ha sido vomitado.

${ }^{6}$ Espíritu que se nutre de alimentos impuros y cadáveres en putrefacción.
}

Metrakshadyotika $a^{7}$, un Chelasaka ${ }^{8}$ etc). Con efecto, en el Libro Segundo del Manava Drama Shastra (Leyes de Manu), la regla $\mathrm{n}^{\circ} 201$ determina que el Dwidja novicio que habla mal de su director (espiritual), se convertirá en asno después de su muerte; si lo calumnia, se tornará perro; si goza de sus bienes sin su permiso, en insecto; si lo mira con ojos envidiosos, en gusano. Otro ejemplo puede ser extraído del Libro Décimo, regla no 91. "Si emplea el sésamo en cualquier otro uso que no sea el de preparar su alimento, untar sus miembros y hacer oblaciones, será reducido al estado de gusano, así como sus abuelos, en los excrementos de un perro". En el Libro Duodécimo encontramos la mayor parte de esas curiosas sanciones (reglas $\mathrm{n}^{\circ} 52-59,61-$ 69 y 71-72), así como advertencias y explicaciones que relacionan la conducta terrenal del hombre, el infierno $^{9}$ (reglas $\mathrm{n}^{\mathrm{o}} 73-75$ ) y las torturas y castigos que sufrirá caso desobedezca las reglas establecidas (reglas $\mathrm{n}^{\circ} 76-78$ ).

Se puede imaginar que este terrorífico escenario ha marcado profundamente el

\footnotetext{
${ }^{7}$ Espíritu que devora materias purulentas.

${ }^{8}$ Genio malo que se alimenta de piojos.

9 Tamisra es la más importante mansión infernal para los hindúes y Asipatravana la selva con hojas de espada, ubicada en pleno infierno. Existen diversos otros lugares de cautiverio y de tortura (regla no 75).
} 
comportamiento de las personas, de modo que no se atrevían a violar las reglas del dharma. En este contexto, la situación de injusticia social era - y aún hoy sigue siendo - generalizada. La ética no puede ser comprendida tal como lo hacen otras religiones, porque el miedo instaurado promueve la consolidación de las castas.

\section{LAS FUENTES BRAHMÁNICAS DEL DERECHO HINDÚ}

Las fuentes brahmánicas de ese peculiar derecho están constituidas por: 1) Vedas, 2) Smritis, 3) costumbres, 4) conciencia individual:

1) Vedas: los Vedas corresponden al mensaje de Dios. Están plasmados en los textos sagrados del hinduismo y se manifiestan en forma de plegarias, himnos o cantos. Sus textos, base de la religión védica, anterior al hinduismo, están constituidos por cuatro escrituras sagradas: a) Rig-veda: colección de himnos dedicados a los dioses (compuestos entre 1700 y 1100 a.C.), b) Sama-veda: asociado a los cánticos devocionales (compuestos aproximadamente en 1700 a.C.), c) Iáyur-veda: asociado al ritual y la liturgia (compuesto entre 1000 y 600 a.C.), d) Átharva-veda: himnos asociados a la cura de enfermedades, larga vida, destrucción de los enemigos etc. (compuestos entre 1200 y 1000 a.C.). Según los estudiosos, parece proceder de una línea de tradiciones independientes de los demás Vedas. Los Vedas marcan esencialmente las normas de conducta. Sus textos son considerados perfectos $\mathrm{y}$ hasta mismos supremos. Están divididos en dos partes: a) Mantras, constituido por palabras de oración y adoración dirigidas al fuego, al viento, al Sol etc., b) Bráhmana, asociado a las explicaciones sobre cómo utilizar adecuadamente los mantras en los rituales.

2) Smrits: los Smrits, según Annoussamy (2001) ${ }^{10}$, son obras del ser humano que tienen por objetivo explicar las reglas de conducta señaladas por los Vedas (aunque su estatus sea similar al de los Vedas). Esa exposición ha sido realizada por los sabios después de la

\footnotetext{
${ }^{10}$ Annoussamy, David. (2001). Le droit indien en
} marche. París: Société de Législation Comparée. 
revelación (smritis). En la

práctica constituyen la principal fuente del derecho brahmánico.

Están divididos en función de su cronología: a) Dharmasutras: han sido redactados en prosa entre los años 800 y 200 a.C. (entre 900 y 300 a.C. para Sarkar, 1958) ${ }^{11}$ y adoptan la forma de aforismos.

Determinan, aunque sin distinciones claras, las obligaciones religiosas, jurídicas y morales derivadas de los Vedas. Tampoco abordan todos los ámbitos del comportamiento humano. Comprenden máximas religiosas y sociales cuya observancia permitiría a la persona adquirir méritos morales que, al lado de la adquisición de bienes (arthasutra, dirigidos al soberano y constituido por directrices de gobierno) y el placer sexual (kamasutra, dirigido a las castas más elevadas), componían los objetivos básicos de la existencia humana, b)

Dharmashastras: redactados a partir de 200 a.C. en versos y

\footnotetext{
${ }^{11}$ Sarkar, U. C. (1958). Epochs in Hindu legal History. Hoshiarpur: Vishveshvaranand Veidc Research Institute.
}

con una exposición más detallada y sistemática. Establecen distinciones entre reglas jurídicas y reglas sociales. El smriti más importante se denomina Manu (Manusmrti), elaborado entre el siglo III a.C. y III d.C. Está compuesto por doce libros que buscan explicar el nacimiento del mundo, las etapas de la vida y el derecho brahmánico. A partir de 700 d.C. numerosos comentarios han sido realizados por los juristas sobre los smritis, que han pasado también a incorporar las costumbres locales. Con el tiempo, cada comunidad pasó a adoptar uno de los comentarios (lo que estaba relacionado con sus propias costumbres locales) como la expresión del derecho vigente que debería regular única y específicamente las relaciones de esa comunidad en concreto. Cada comentario, por su vez, ha servido de origen para numerosas escuelas doctrinales. Por último, importa mencionar la existencia de los Nibandha, explicaciones redactadas durante la larga transición del derecho brahmánico originario hacia el derecho misto indo-islámico 
introducido por los musulmanes.

3) Costumbres: para ser considerada como fuente del derecho, la costumbre debe cumplir tres requisitos: 1) ser antigua (la jurisprudencia india actual determina que es antigua la costumbre aplicada por más de un siglo), 2) ser considerada obligatoria por la comunidad, 3) no ir en contra del orden público. Las costumbres, muy numerosas, eran - y son aún específicas de cada lugar $\mathrm{y}$ variables en función de las castas de las personas. En otras palabras, la costumbre no solamente varía en función de la casta como también en función de la comunidad o de los diferentes colectivos existentes en la comunidad. Existe una enorme mezcla de costumbres locales y costumbres comunes (menos expuestos a variaciones locales). Caso no existiera una costumbre común, sería de aplicación obligatoria los dharmashastras. Según David \& Jauffret-Spinosi (2002) ${ }^{12}$ la costumbre tiene un valor

12 David, René \& Jauffret-Spinosi, Camille. (2002). Les grands systèmes de droit contemporains (11ème edition). Paris: DallozSirey. superior al derecho sagrado. Efectivamente, todos los dharmashastras reconocen ese estatus a las tradiciones, así como el smriti narada declara que la costumbre decidirá sobre todo, pudiendo hasta mismo descartar la ley sagrada. La costumbre, sin embargo, no debe ser vista como un elemento de oposición a la ley sagrada, pero de complementación. Además, como señala Annoussamy $(2001)^{13}$, es muy probable que las leyes sagradas tengan sido elaboradas con base en las costumbres vigentes en la época de su elaboración. A propósito, las costumbres no pueden ser revocadas por el legislador indio (en la India actual se ha intentado revocar ciertas tradiciones milenarias pero sin cualquier éxito).

4) $\mathrm{La}$ conciencia individual: el arbitrio de una persona para decidir si una determinada conducta es o no la adecuada, en función de los intereses de la comunidad (tanto la general como de la casta, subcasta o intercasta a que pertenece y de

\footnotetext{
${ }^{13}$ Annoussamy, David. (2001). Le droit indien en marche. París: Société de Législation Comparée.
} 
su particular visión del hinduismo) es una fuente del derecho brahmánico absolutamente inconcebible para el derecho occidental. Aunque existan expectativas generales relacionadas al comportamiento personal y su sumisión al interés común de la colectividad, ningún otro sistema jurídico consagra la conciencia individual como fuente del derecho. No obstante, como veremos, la conciencia individual ejerce un rol muy relevante en la asunción de compromisos y cumplimiento de deberes en el ámbito del sintoísmo.

También se podría introducir como fuente del derecho hindú los precedentes judiciales típicos del Commom Law (aunque sin la fuerza consagrada por los británicos). Sin embargo, más que una fuente, los precedentes funcionan en la India actual principalmente como un método de apreciación y decisión judicial, incluyendo los casos en que el derecho islámico o brahmánico constituyen el tema central de la controversia judicial.

En India, el compromiso estatal de dotar a los ciudadanos de un código civil uniforme (previsto en el artículo 44 de la constitución del país) colisiona directamente con el denominado "estatuto personal" (derecho confesional). Esos estatutos personales se encuentran así, regulados por la confesión religiosa de la persona. Están reconocidas las siguientes religiones, además del hinduismo: el cristianismo, el judaísmo, el islamismo y el zoroastrismo. Sin embargo, según Cuniberti (2011) ${ }^{14}$ el derecho hindú se aplica también a los sikhs, budistas y jaïns, así como a las personas que no practiquen cualquier religión o que practiquen alguna religión que no sea reconocida por el derecho personal.

\section{LA ÉTICA FINANCIERA EN EL MANUSMRTI}

El tema de la ética financiera se encuentra principalmente en las denominadas Leyes de Мапи 15 (Manusmrti) ${ }^{16}$ un importante texto de la antigua sociedad india transmitida por Manu hacia los rishis (grandes sabios de la antigüedad védica, como Kasiapa, Vishuámitra o Vásista) que le habían pedido que los iluminara. Las Leyes de

\footnotetext{
${ }^{14}$ Cuniberti, Gilles. (2011). Grands systèmes de droit contemporains (2e édition). Paris: LGDJ; Edición.

${ }^{15}$ Manu es considerado el antepasado común de toda la humanidad.

${ }^{16}$ Su datación es incierta, siendo la posición más común entre el siglo III a.C. y III d.C.
} 
Manu enfatizaban el dharma sin vincularse a los Vedas. Esas leyes constituyen uno de los dieciocho smritis del Dharmashastra. Contiene cientos de versos que presentan reglas y códigos de conducta aplicables a los individuos y a la sociedad. Sobre el dinero, por ejemplo, encontramos disposiciones en el Libro Cuarto (regla $n^{\circ}$ 248: voluntariedad en la entrega de la limosna) y en el Libro Séptimo (regla $\mathrm{n}^{\circ}$ 124: conducta del rey ante la extorsión cometida por funcionarios públicos $\mathrm{y}$ empleados en general). Sobre los préstamos con intereses, por su vez, son aplicables las reglas contenidas en el Libro Octavo. Destacamos las siguientes:

No 140. "El que prestó dinero, si tiene una prenda debe recibir, además de su capital, el interés fijado por Vasishtha; es decir, la octogésima parte de ciento por más o uno y cuarto".

$\mathrm{N}^{\circ}$ 141. "O que, si no tiene prenda, tome dos por ciento al mes, recordando el deber de las gentes de bien; pues tomando dos por ciento no es culpable de ganancias ilícitas”.

El porcentaje de interés está vinculado a la casta, como señala la regla $\mathrm{n}^{\circ}$ 142: "Que reciba dos por ciento como interés mensual (nunca más) de un
Bracmán, tres de un Chatrya, cuatro de un Vaisya y cinco de un Sudra, siguiendo el orden directo de clases". Por su vez, la regla $n^{\circ} 143$ establece que: “...si le han entregado un terreno o una vaca como prenda, con el permiso de aprovecharse de ella, no debe recibir otro interés por la suma prestada y después de un gran lapso de tiempo, o cuando las ganancias suben hasta el valor de la deuda, no puede dar esta prenda ni venderla”. Otras reglas destacables son: $n^{\circ} 144$ (usufructo sin permiso del propietario de ciertos bienes: vestidos, adornos y otros objetos de la misma clase), no 158 ("El hombre que se hace fiador de la comparecencia de un deudor y que no puede presentarlo debe pagar por entero la deuda con su propio haber"), $\mathrm{n}^{\circ} 159$ (imposibilidad de trasmitir ciertas clases de deudas a los hijos: fianza, dinero prometido sin motivo a cortesanas o a músicos, deuda de juegos, de licores espirituosos y restos de pagos de multas o impuestos), $\mathrm{n}^{\mathrm{o}}$ 160-162 (reglas especiales sobre la fianza), no 166 (pago de deudas por los parientes del prestatario fallecido). Muy interesante es la regla $n^{\circ} 193$ sobre la apropiación indebida, al que transcribimos por completo:

$\mathrm{N}^{\circ}$ 193. "El que con falsos 
ofrecimientos de servicios se apodera del dinero ajeno, debe padecer públicamente, así como sus cómplices, diversas clases de suplicios, según las circunstancias, y aún la muerte”.

Además de la diferencia establecida en relación a los intereses, existen otras reglas que marcan fuertemente la diferencia entre las castas. La regla $\mathrm{n}^{\circ}$ 410, por ejemplo, determina "Que el rey ordena a los Vaisyas comerciar, prestar dinero a crédito, labrar la tierra, o criar ganados; que a los Sudras, les ordene servir a los Dwidjas $^{17}$ ”. Por el mismo camino siguen las reglas $n^{\circ}$ 411- 414. Destaco, por su contundencia, las reglas:

$\mathrm{N}^{\mathrm{o}}$ 413. "Pero el que obligue a un Sudra, haya o no sido comprado, a desempeñar funciones serviles, pues fue creado por el Ser existente por sí mismo para servir a los Bracmanes".

No 414. "Un Sudra, aunque haya sido libertado por su amo no se liberta del estado de servidumbre; pues siéndole natural tal estado, ¿quién podría eximirlo?"

\footnotetext{
${ }^{17}$ Los Dwidjas ("lo que es dos veces nascido" o "renacido de las entrañas de sí mismo") corresponden a los denominados proyectores conscientes. Son hombres espirituales, anteriormente humanos comunes que han logrado superar los límites del propio cuerpo físico.
}

Sin embargo, las sanciones son aplicables con una severidad inversa a la importancia de las castas en la estructura social india, en función del grado de discernimiento asociado al nivel sociocultural de la persona. Así,

No 337. "La multa de un Sudra por cualquier robo debe ser ocho veces más considerable que la pena ordinaria; la de un Vaisya, dieciséis veces; la de un Chatrya, treinta y dos veces".

$\mathrm{N}^{\mathrm{o}}$ 338. "La de un Bracmán sesenta y cuatro veces o cien veces o ciento veintiocho veces más considerable, cuando cada uno de ellos conoce perfectamente la bondad o la maldad de sus actos".

El Libro Décimo del Manusmrit dispone que el Bracmán y el Chatrya solo pueden excepcionalmente prestar a crédito (regla $n^{\circ} 117$ ). La regla $n^{\circ} 120$ procura establecer una situación de justicia fiscal: "El impuesto sobre la clase comerciante, que en épocas de prosperidad sólo llega a la dozava parte de las cosechas y al quincuagésimo de los beneficios pecuniarios, puede ser en los casos de apuro la octava y aún la cuarta parte de las cosechas y la vigésima de las ganancias en dinero; los Sudras, los obreros y los artesanos deben concurrir 
con su trabajo y no pagar impuesto alguno”.

La distinción entre castas es nuevamente exaltada en este Libro. Con efecto, la regla $n^{\circ} 121$ proclama que el Shudrá debe tener por objetivo servir a las demás castas, obteniendo el Cielo caso su amo sea un Brahmán ( $\mathrm{n}^{\circ}$ 122). La regla n 123 complementa: "Servir a los Bracmanes es la acción más digna de alabanza en un Sudra; cualquiera otra cosa que pueda hacer no le trae recompensa". Por su vez, el Brahmán debe tomar en cuenta la habilidad, el celo y el número de personas sostenidas por el Shudrá ( $n^{\circ}$ 124) antes de aceptarlo como sirviente. Caso lo haga, tendrá que proporcionarle medios de vida suficientes. El Shudrá no puede acumular riquezas, aun cuando esté en la posibilidad de hacerlo, en virtud de una supuesta predisposición a la insolencia ( $\left.n^{\circ} 129\right)$. Más adelante, en el Libro Octavo, se advierte sobre la miseria que se abatiría sobre un reino poblado por muchos Shudrás $\left(n^{\circ} 22\right)$ o en que ellos puedan emitir juicios de valor delante del rey $\left(\mathrm{n}^{\mathrm{o}} 21\right)$. La situación de injusticia a que se ven sometidos los Shudrás, los Dalits y otros grupos es palpable bajo el brahmanismo, según la perspectiva occidental. Sin embargo, son los propios individuos componentes de cada casta los que se rebelan contra la igualdad entre todos ellos. Las razones son distintas e inversas. Por un lado, las castas más elevadas temen perder su status social. Por otro, tanto los Shudrás como los descastados Dalits se consideran merecedores de la situación precaria en que viven, como resultado de las conductas practicadas en encarnaciones anteriores. Al considerar la precariedad y la injusticia una forma kármica de expiación, temen que igualar sus derechos les hará repetir el mismo rol (condición de Shudrá o Dalit) o rebajarlos a la condición de ciertos animales en sus futuras reencarnaciones. Concretamente sobre las deudas, las únicas reglas previstas en las Leyes de Manu (Libro Octavo) son:

$\mathrm{N}^{\mathrm{o}}$ 47. "Que cuando un acreedor viene a intentar una demanda ante él, para el recobro de una suma prestada que retiene un deudor, haga pagar al deudor después de que el acreedor ha probado la deuda”.

$\mathrm{N}^{\circ}$ 48. "Para forzar a su deudor a que cumpla, puede recurrir un acreedor a los diferentes medios que están en uso para cobrar la deuda”.

$\mathrm{N}^{\mathrm{o}}$ 49. "Por medios conformes al deber moral, por medio de proceso por 
astucia, por la miseria y con medidas violentas puede un acreedor hacerse pagar la suma que le adeudan”.

De acuerdo con Burnell $(2013)^{18}$, el deber moral debe ser reforzado a través de la mediación de amigos y parientes. No obstante, también se contempla la posibilidad de seguir al deudor por todas partes y hasta de permanecer en su casa, todo con el propósito de obligársele a pagar su deuda. La presión puede incluir lo que la regla denomina "miseria" o presión económica. Esta forma de cobro es considerada compatible con el deber moral. Por otro lado, la "astucia" está asociada a la toma de un objeto o animal del deudor (o su retención, caso depositado por el deudor) como forma de presionarlo a pagar su deuda. La astucia configura un "fraude legal". La regla incorpora también, de modo equiparable al fraude legal, la adopción de medidas violentas. Kosambi (1994 ${ }^{19}$ señala que son consideradas legales ("obligaciones legales", según el autor): a) el cautiverio del hijo, de la mujer o del ganado del deudor para presionarle ("obligarle") a pagar su deuda o b) la vigilancia continua del acreedor en la puerta de la

18 Burnell, A. C. (2013). The Ordinances of Manu. London: Routledge.

19 Kosambi, D. D. (1994). The culture and civilization of ancient India in historical outline. Pradesh: Vikas Publishing House Pvt Ltd. casa del deudor. El modo violento de cobro incluiría, por ejemplo, atar al deudor (llevándolo a la casa del acreedor) y pegarle (o emplear "medios similares") para obligarle a pagar su deuda. Esta violencia está justificada y es considerada legal.

En el Libro Octavo del Manava Drama Shastra encontramos otras reglas interesantes: $n^{\circ} 50$ (devolución forzosa del bien prestado por el acreedor al deudor), $\mathrm{n}^{\mathrm{o}} 51$ (sanción pecuniaria aplicada al deudor que niega la existencia de una deuda probada por el acreedor), $\mathrm{n}^{\mathrm{o}} 52$ (testimonios o otras pruebas contra el deudor que niega la existencia de una deuda ante los tribunales), $\quad \mathrm{n}^{\mathrm{o}} 139 \quad$ (sanciones pecuniarias al deudor en caso de reconocimiento y denegación de la deuda), $n^{\circ} 144$ (prohibición de dar en usufructo, sin permiso del propietario, ciertos bienes depositados), $n^{o} 150$ (reparación de usufructo ilegítimo). Las reglas siguientes $\left(\mathrm{n}^{\circ} 151,152,153,154\right.$, 155,176 y 177) son muy relevantes y merecen ser transcritas:

$\mathrm{N}^{\circ}$ 151. "El interés de una suma prestada, recibido de una sola vez y no por mes o por día, no debe sobrepasar al doble de la deuda; es decir, no debe ascender a una suma mayor que el capital que se reembolsa al mismo 
tiempo, y tratándose del grano, de los frutos, de la lana o de la crin, de las bestias de carga, prestados para ser pagados en objetos del mismo valor, el interés debe cuando más elevarse hasta quintuplicar la deuda”.

$\mathrm{N}^{\circ}$ 152. "Un interés que sobrepasa la tasa legal y que se aparta de la regla precedente, no es válido; los Sabios lo llaman procedimiento usurario; el que presta no debe recibir, cuanto más, sino cinco por ciento".

No 153. "Que el que presta por un mes o por dos o por tres, a cierto interés, no reciba el mismo interés por más de un año, ni ningún interés desaprobado, ni el interés del interés, por convención previa, ni un interés arrancado a un deudor en un momento de apuro, ni ganancias exorbitantes de una prenda cuyo usufructo reemplaza al interés”.

$\mathrm{N}^{\circ}$ 154. "El que no pueda pagar una deuda al tiempo fijado y que desea renovar el contrato, puede hacerlo de nuevo con el asentimiento del que prestó, pagando todo el interés debido".

$\mathrm{N}^{\mathrm{o}}$ 155. "Pero si por algún golpe de la suerte se encuentra en la imposibilidad de ofrecer el pago del interés, puede inscribir como capital en el contrato que renueva el interés que hubiera debido pagar”.

No 176. “El deudor que imaginándose que tiene una gran influencia con el soberano, viene a quejarse ante el príncipe de que su acreedor trata de recobrar por los medios permitidos lo que se le debe, debe ser obligado por el rey a pagar como multa la cuarta parte de la suma y a devolver al acreedor lo que le debe”.

No 177. "Un deudor puede pagar a su acreedor por medio de su trabajo si es la misma clase o de una clase inferior; pero que si es de una clase superior, pague la deuda poco a poco, según sus facultades".

\section{CONCLUSIONES}

Como vimos, las Leyes de Manu buscan regular los intereses, fijándoles ciertos parámetros y límites. Aunque varias de esas reglas puedan considerarse modernas para su época $y$, en muchos aspectos, aplicables hasta hoy, en la práctica, el tema de la ética sigue siendo extremadamente complejo en una sociedad donde se considera natural la existencia de seres serviles, de "intocables", de "invisibles" (personas que solamente están autorizadas a salir por la noche, bajo pena de encarcelamiento hasta la muerte por inanición) y de niñas devadasi 
(compelidas por la tradición religiosa a satisfacer sexualmente a cualquier hombre del pueblo, sin derecho a negarse). En una sociedad que ampara, promueve y justifica la división social en castas con base en escrituras sagradas (como el himno Púrusha-sukta del Rig-veda), cualquier discusión profunda sobre ética, bajo la perspectiva occidental, tenderá a ser diluida (Frawley, 2001 ${ }^{20}$. Con efecto, los textos sagrados señalan que a partir de la inmolación de Púrusha, Atman o Brahman (el primero y supremo ser) han surgido las cuatro grandes castas: a) los Brahmanes (salidos de su boca), b) los Chatrías (salidos de sus hombros), c) los Vaishias (salidos de sus caderas), c) los Shudrás (salidos de sus pies). Los descastados Dalits, por su vez, proceden del polvo dejado por los pies de Dios. La moral hinduista está basada en la idea de que cada casta tiene su propio conjunto de deberes. En un sentido espiritual, cada uno representa y posee un dharma, que debe ser escrupulosamente observado para que el alma, después de la muerte física, afronte su propio karma (destino), transmigrando a un cuerpo inferior $\mathrm{o}$ superior en función de los actos

\footnotetext{
${ }^{20}$ Frawley, D. (2001). The Rig Veda and the History of India: Rig Veda Bharata Itihasa. New Delhi: Aditya Prakashan.
}

practicados en vida. La desobediencia o no acatamiento de las reglas consagradas, por ejemplo, en las Leyes de Manu, harán con que la transmigración kármica resulte en el nacimiento (reencarnación) en una casta inferior, en el cuerpo de un animal o en ciertas plantas (matorral, lianas). Al contrario, creen que todo aquél que obedece resignada y fielmente su dharma reencarnará en la siguiente casta superior. No se puede hablar en ética, financiera o no, según la perspectiva occidental, que sea generalizada y uniforme para todas las castas, intercastas, subcastas e individuos descastados en India. Su estructura social y el valor transcendental atribuido a costumbres $\mathrm{y}$ tradiciones antiquísimas con valor superior a las escrituras sagradas, cristalizan un conjunto distinto de derechos y deberes entre las personas en función de su pertenencia a una $\mathrm{u}$ otra categoría social. En el caso de los descastados Dalits, hablar en ética financiera o mismo general es sumamente complicado. $\mathrm{Su}$ participación en transacciones financieras es prácticamente inexistente. Las que eventualmente surjan estarían sometidas a las costumbres locales, del poblado en que esté ubicado el individuo. 


\section{REFERENCIAS BIBLIOGRÁFICAS}

Annoussamy, David. (2001). Le droit indien en marche. París: Société de Législation Comparée.

Burnell, A. C. (2013). The Ordinances of Manu. London: Routledge.

Cuniberti, Gilles. (2011). Grands systèmes de droit contemporains (2e édition). Paris: LGDJ; Edición.

David, René \& Jauffret-Spinosi, Camille. (2002). Les grands systèmes de droit contemporains (11ème edition). Paris: Dalloz-Sirey.

Frawley, D. (2001). The Rig Veda and the History of India: Rig Veda Bharata Itihasa. New Delhi: Aditya Prakashan. Kosambi, D. D. (1994). The culture and civilization of ancient India in historical outline. Pradesh: Vikas Publishing House Pvt Ltd.

Mayne, John Dowson. (1953). Treatise on Hindu Law and Usage. Madras: Higginbothams.

Sarkar, U. C. (1958). Epochs in Hindu legal History. Hoshiarpur: Vishveshvaranand Veidc Research Institute.
Viswanatha, S. V. (2013). Racial synthesis in Hindu culture ( $\mathrm{n}^{\mathrm{o}}$ 47). London: Routledge. 